\title{
Model and Extended Kuhn-Tucker Approach for Bilevel Multi-follower Decision Making in a Referential-Uncooperative Situation
}

Jie Lu, Chenggen Shi, Guangquan Zhang \& Tharam Dillon

\author{
Faculty of Information Technology \\ University of Technology, Sydney \\ Sydney, NSW 2007, Australia \\ Phone: 6102 95144534, Fax: 610295144535 \\ Email: $\{$ jielu, cshi, zhangg, tharam\}@it.uts.edu.au
}

\begin{abstract}
When multiple followers are involved in a bilevel decision problem, the leader's decision will be affected, not only by the reactions of these followers, but also by the relationships among these followers. One of the popular situations within this framework is where these followers are uncooperatively making decisions while having cross reference of decision information. This situation is called a referential-uncooperative situation in this paper. The well-known Kuhn-Tucker approach has been successfully applied to a oneleader-and-one-follower linear bilevel decision problem. This paper extends this approach to deal with the above-mentioned linear referential-uncooperative bilevel multifollower decision problem. The paper first presents a decision model for this problem. It then proposes an extended Kuhn-Tucker approach to solve this problem. Finally, a numeric example illustrates the application of the proposed Kuhn-Tucker approach.
\end{abstract}

Keywords: bilevel programming, bilevel multi-follower decision, Kuhn-Tucker approach, optimization

\section{Introduction}

In a bilevel programming (BLP) problem, the leader cannot completely control his/her follower but is influenced by the reaction of his/her follower. Such a situation is appearing in decision making of many decentralized organizations. BLP was motivated by the game theory of Von Stackelberg [1] in the context of unbalanced economic 
markets [2]. The majority of research on BLP has centered on the linear version of the problem in which only one follower is involved. There have been nearly two dozen algorithms, such as, the $K^{\text {th }}$ best approach [3, 4], Kuhn-Tucker approach [5-7], complementarity pivot approach [8], penalty function approach [9-13], proposed for solving linear BLP problems since the field being caught the attention of researchers in the mid-1970s. Kuhn-Tucker approach has been proven to be a valuable analysis tool with a wide range of successful applications for linear BLP [2, 6, 7, 14-16].

Although much research has been carried out in the area, the existing bilevel technology has mainly limited to a specific situation comparing one leader and one follower. However, in a real-world bilevel decision problem, the lower level of a bilevel decision may involve multiple decision units. For example, the dean of a faculty is the leader, and all the heads of departments in the faculty are the followers in making a faculty annual budget. The leader (the dean, for example)'s decision will be affected, not only by the reactions of the multiple followers (these heads of departments in the faculty), but also by the relationships among these followers. Each of the leader's possible decisions is influenced by the various reactions of his/her followers who may have had a share in decision information, objectives and constraints. Hence, a bilevel multi-follower (BLMF) decision problem is a common case in any organizational decision practice, and involves various different decision situations.

Our previous work [17-20] presented overcame some fundamental deficiency of existing linear BLP theory. Based on that, we have recently generalized a framework for BLMF decision problems, and identified nine main kinds of relationships amongst these followers [21]. The uncooperative model is the most popular situation for BLMF decision problems. This model handles the case in which there is no shared decision variable among the followers. Under this uncooperative model, the most basic situation is that any follower also doesn't make any reference to other followers' decision. For a model and related approaches in finding an optimal solution for this particular decision situation, the reader is referred to [21-23]. Another such uncooperative situation is that though these followers are uncooperative (no sharing of decision variables) but have cross reference of information by considering other followers' decision results in each of their own decision 
objective and constraint. We call this case as a referential-uncooperative situation, and this paper will particularly focus on this situation.

Following the introduction, this paper proposes a model for linear BLMF decision making in a referential-uncooperative situation in Section 2. An extended Kuhn-Tucker approach for solving this model is presented in Section 3. A numeric example for this approach is illustrated in Section 4. Further remarks are concluded in Section 5.

\section{The linear BLMF decision model in a referential-uncooperative situation}

Under the BLMF framework, if two followers don't have any shared decision variable, it is called an uncooperative relationship. But if one of them has a reference of another follower's decision information in his/her objective or constraint, the two followers are defined having a referential-uncooperative relationship. When there is a referentialuncooperative relationship in a BLMF model, this model is called a referentialuncooperative BLMF model. We present this model as follows.

For $x \in X \subset R^{n}, y_{i} \in Y_{i} \subset R^{m_{i}}, F: X \times Y_{1} \times \ldots \times Y_{K} \rightarrow R^{1}$, and $f_{i}: X \times Y_{1} \times \ldots \times Y_{K} \rightarrow R^{1}$, $i=1,2, \ldots, K$, a linear BLMF decision problem where $K(\geq 2)$ followers are involved and there are not shared decision variables, but shared information in objective functions and constraint functions among the followers is defined as follows:

$$
\begin{aligned}
& \min _{x \in X} F\left(x, y_{1}, \ldots, y_{K}\right)=c x+\sum_{s=1}^{K} d_{s} y_{s} \\
& \text { subject to } A x+\sum_{s=1}^{K} B_{s} y_{s} \leq b \\
& \min _{y_{i} \in Y_{i}} f_{i}\left(x, y_{1}, \ldots, y_{K}\right)=c_{i} x+\sum_{s=1}^{K} e_{i s} y_{s} \\
& \text { subject to } A_{i} x+\sum_{s=1}^{K} C_{i s} y_{s} \leq b_{i},
\end{aligned}
$$


where $c \in R^{n}, c_{i} \in R^{n}, d_{i} \in R^{m_{i}}, e_{i s} \in R^{m_{s}}, b \in R^{p}, b_{i} \in R^{q_{i}}, A \in R^{p \times n}, B_{i} \in R^{p \times m_{i}}$, $A_{i} \in R^{q_{i} \times n}, C_{i s} \in R^{q_{i} \times m_{s}}, i, s=1,2, \ldots, K$.

Definition 1 A compact set is compact if every open cover of the entire space has a finite subcover. For example, $[a, b]$ is compact in $R$ (the Heine-Borel theorem) [24].

Corresponding to (1), we give following basic definition.

\section{Definition 2}

(a) Constraint region:

$$
\begin{aligned}
& S=\left\{\left(x, y_{1}, \ldots, y_{K}\right) \in X \times Y_{1} \times \ldots \times Y_{k}, A x+\sum_{s=1}^{K} B_{s} y_{s} \leq b,\right. \\
& \left.A_{i} x+\sum_{s=1}^{K} C_{i s} y_{s} \leq b_{i}, i=1,2, \ldots, K\right\} .
\end{aligned}
$$

The constraint region refers to all possible combinations of choices that the leader and followers may make.

(b) Projection of $S$ onto the leader's decision space:

$$
S(X)=\left\{x \in X: \exists y_{i} \in Y_{i}, A x+\sum_{s=1}^{K} B_{s} y_{s} \leq b, A_{i} x+\sum_{s=1}^{K} C_{i s} y_{s} \leq b_{i}, i=1,2, \ldots, K\right\} .
$$

(c) Feasible set for each follower $\forall x \in S(X)$ :

$$
S_{i}(x)=\left\{y_{i} \in Y_{i}:\left(x, y_{1}, \ldots, y_{K}\right) \in S\right\} .
$$

The feasible region for each follower is affected by the leader's choice of $x$, and the allowable choices of each follower are the elements of $S$.

(d) Each follower's rational reaction set for $x \in S(X)$ :

$$
P_{i}(x)=\left\{y_{i} \in Y_{i}: y_{i} \in \arg \min \left[f_{i}\left(x, \hat{y}_{i}, y_{j}, j=1,2, \ldots, K, j \neq i\right): \hat{y}_{i} \in S_{i}(x)\right]\right\},
$$

where $i=1,2, \ldots, K, \arg \min \left[f_{i}\left(x, \hat{y}_{i}, y_{j}, j=1,2, \ldots, K, j \neq i\right): \hat{y}_{i} \in S_{i}(x)\right]=$

$$
\left\{y_{i} \in S_{i}(x): f_{i}\left(x, y_{1}, \ldots, y_{K}\right) \leq f_{i}\left(x, \hat{y}_{i}, y_{j}, j=1,2, \ldots, K, j \neq i\right), \hat{y}_{i} \in S_{i}(x)\right\} . \text { The }
$$

followers observe the leader's action and simultaneously react by selecting $y_{i}$ from their feasible set to minimize their objective functions.

(e) Inducible region:

$$
I R=\left\{\left(x, y_{1}, \ldots, y_{K}\right):\left(x, y_{1}, \ldots, y_{K}\right) \in S, y_{i} \in P_{i}(x), i=1,2, \ldots, K\right\} .
$$

Thus in terms of the above notations, (1) can be written as 


$$
\min \left\{F\left(x, y_{1}, \ldots, y_{K}\right):\left(x, y_{1}, \ldots, y_{K}\right) \in I R\right\}
$$

We propose the following theorem to characterize the condition under which there is an optimal solution for (1).

Theorem 1 If $S$ is nonempty and compact, there exists an optimal solution for a linear BLMFP problem.

Proof: Since $S$ is nonempty, there exist a point $\left(x^{*}, y_{1}^{*}, \ldots, y_{K}^{*}\right) \in S$. Then, we have

$$
x^{*} \in S(X) \neq \phi,
$$

by Definition 2(b). Consequently, we have

$$
S_{i}\left(x^{*}\right) \neq \phi, i=1,2, \ldots, K,
$$

by Definition 2(c). Because $S$ is compact and Definition 2(d), we have

$$
\begin{aligned}
P_{i}\left(x^{*}\right)= & \left\{y_{i} \in Y_{i}: y_{i} \in \arg \min \left[f_{i}\left(x^{*}, \hat{y}_{i}, y_{j}, j=1,2, \ldots, K, j \neq i\right): \hat{y}_{i} \in S_{i}\left(x^{*}\right)\right]\right\} \\
= & \left\{y_{i} \in Y_{i}: y_{i} \in\left\{y_{i} \in S_{i}\left(x^{*}\right):\right.\right. \\
& \left.\left.f_{i}\left(x^{*}, y_{1}, \ldots, y_{K}\right) \leq f_{i}\left(x^{*}, \hat{y}_{i}, y_{j}, j=1,2, \ldots, K, j \neq i\right), \hat{y}_{i} \in S_{i}\left(x^{*}\right)\right\}\right\} \neq \phi,
\end{aligned}
$$

where $i=1,2, \ldots, K$. Hence, there exists $y_{i}^{0} \in P_{i}\left(x^{*}\right), i=1,2, \ldots, K$ such that $\left(x^{*}, y_{1}^{0}, \ldots, y_{K}^{0}\right) \in S$. Therefore, we have

$$
I R=\left\{\left(x, y_{1}, \ldots, y_{K}\right):\left(x, y_{1}, \ldots, y_{K}\right) \in S, y_{i} \in P_{i}(x), i=1,2, \ldots, K\right\} \neq \phi,
$$

by Definition 2(e). Because we are minimizing a linear function

$\min _{x \in X} F\left(x, y_{1}, \ldots, y_{K}\right)=c x+\sum_{s=1}^{K} d_{s} y_{s}$ over $I R$, which is nonempty and bounded, an optimal solution to the linear BLMFP problem must exist. So the proof is completed.

\section{An extended Kuhn-Tucker approach}

Let write a linear programming (LP) as follows.

$$
\begin{aligned}
& \min f(x)=c x \\
& \text { subject to } A x \geq b \\
& \qquad x \geq 0,
\end{aligned}
$$

where $c$ is an n-dimensional row vector, $b$ an m-dimensional column vector, $A$ an $m \times n$ matrix with $m \leq n$, and $x \in R^{n}$. 
Let $\lambda \in R^{m}$ and $\mu \in R^{n}$ be the dual variables associated with constraints $A x \geq b$ and $x \geq 0$, respectively. Bard [2] gave the following proposition.

Proposition 1 A necessary and sufficient condition that $\left(x^{*}\right)$ solves above LP is that there exist (row) vectors $\lambda^{*}, \mu^{*}$ such that $\left(x^{*}, \lambda^{*}, \mu^{*}\right)$ solves:

$$
\begin{aligned}
& \lambda A-\mu=-c \\
& A x-b \geq 0 \\
& \lambda(A x-b)=0 \\
& \mu x=0 \\
& x \geq 0, \lambda \geq 0, \mu \geq 0 .
\end{aligned}
$$

Proof: (See reference [2] PP. 59-60)

Let $u_{i} \in R^{p}, v_{i} \in R^{q_{1}+q_{2}+\ldots+q_{K}}$ and $w_{i} \in R^{m_{i}}(i=1,2, \ldots, K)$ be the dual variables associated with constraints $\left(A x+\sum_{s=1}^{K} B_{s} y_{s} \leq b\right),\left(A^{\prime} x+\sum_{s=1}^{K} C_{s}^{\prime} y_{s} \leq b^{\prime}\right)$ and $y_{i} \geq 0 \quad(i=1, \ldots, K)$, respectively, where $A^{\prime}=\left(A_{1}, A_{2}, \ldots, A_{K}\right)^{T}, C_{i}^{\prime}=\left(C_{i 1}, C_{i 2}, \ldots, C_{i K}\right)^{T}, b^{\prime}=\left(b_{1}, b_{2}, \ldots, b_{K}\right)^{T}$. We have a following theorem.

Theorem 2 A necessary and sufficient condition that $\left(x^{*}, y_{1}^{*}, \ldots, y_{K}^{*}\right)$ solves the linear BLMFP problem (1) is that there exist (row) vectors $u_{1}^{*}, u_{2}^{*}, \ldots, u_{K}^{*}, v_{1}^{*}, v_{2}^{*}, \ldots, v_{K}^{*}$ and $w_{1}^{*}, w_{2}^{*}, \ldots, w_{K}^{*}$ such that $\left(x^{*}, y_{1}^{*}, \ldots, y_{K}^{*}, u_{1}^{*}, \ldots, u_{K}^{*}, v_{1}^{*}, \ldots, v_{K}^{*}, w_{1}^{*}, \ldots, w_{K}^{*}\right)$ solves:

$$
\begin{array}{r}
\min _{x \in X} F\left(x, y_{1}, \ldots, y_{K}\right)=c x+\sum_{s=1}^{K} d_{s} y_{s} \\
\text { subject to } A x+\sum_{s=1}^{K} B_{s} y_{s} \leq b \\
A^{\prime} x+\sum_{s=1}^{K} C_{s}^{\prime} y_{s} \leq b^{\prime} \\
u_{i} B_{i}+v_{i} C_{i}^{\prime}-w_{i}=-e_{i i}
\end{array}
$$




$$
\begin{gathered}
u_{i}\left(b-A x-\sum_{s=1}^{K} B_{s} y_{s}\right)+v_{i}\left(b^{\prime}-A^{\prime} x-\sum_{s=1}^{K} C_{s}^{\prime} y_{s}\right)+w_{i} y_{i}=0 \\
x \geq 0, y_{j} \geq 0, u_{j} \geq 0, v_{j} \geq 0, w_{j} \geq 0, j=1,2, \ldots, K,
\end{gathered}
$$

where $i=1,2 \ldots, K$.

\section{Proof:}

(1) Let us get an explicit expression of (2).

Rewrite (2) as follows:

$$
\begin{aligned}
& \min F\left(x, y_{1}, \ldots, y_{K}\right) \\
& \text { subject to }\left(x, y_{1}, \ldots, y_{K}\right) \in I R .
\end{aligned}
$$

We have

$$
\begin{gathered}
\min F\left(x, y_{1}, \ldots, y_{K}\right) \\
\text { subject to }\left(x, y_{1}, \ldots, y_{K}\right) \in S \\
y_{i}=P_{i}(x),
\end{gathered}
$$

where $i=1,2, \ldots, K$, by Definition $2(\mathrm{e})$. Then, we have

$\min F\left(x, y_{1}, \ldots, y_{K}\right)$

subject to $\left(x, y_{1}, \ldots, y_{K}\right) \in S$

$$
y_{i} \in \arg \min \left[f_{i}\left(x, \hat{y}_{i}, y_{j}, j=1,2, \ldots, K, j \neq i\right): \hat{y}_{i} \in S_{i}(x)\right],
$$

where $i=1,2, \ldots, K$, by Definition $2(\mathrm{~d})$. We rewrite it as:

$\min F\left(x, y_{1}, \ldots, y_{K}\right)$

subject to $\left(x, y_{1}, \ldots, y_{K}\right) \in S$

$\min f_{i}\left(x, y_{1}, \ldots, y_{K}\right)$

subject to $y_{i} \in S_{i}(x)$,

where $i=1,2, \ldots, K$. We have

$\min F\left(x, y_{1}, \ldots, y_{K}\right)$

subject to $\left(x, y_{1}, \ldots, y_{K}\right) \in S$

$$
\begin{aligned}
& \min _{y_{i} \in Y_{i}} f_{i}\left(x, y_{1}, \ldots, y_{K}\right) \\
& \text { subject to }\left(x, y_{1}, \ldots, y_{K}\right) \in S,
\end{aligned}
$$


where $i=1,2, \ldots, K$, by Definition 2(c). Consequently, we can have

$$
\begin{aligned}
& \min _{x \in X} F\left(x, y_{1}, \ldots, y_{K}\right)=c x+\sum_{s=1}^{K} d_{s} y_{s} \\
& \text { subject to } A x+\sum_{s=1}^{K} B_{s} y_{s} \leq b \\
& A_{j} x+\sum_{s=1}^{K} C_{i s} y_{s} \leq b_{i}, j=1,2, \ldots, K \\
& \min _{y_{i} \in Y_{i}} f_{i}\left(x, y_{i}, \ldots, y_{K}\right)=c_{i} x+\sum_{s=1}^{K} e_{i s} y_{s} \\
& \text { subject to } A x+\sum_{s=1}^{K} B_{s} y_{s} \leq b \\
& A_{j} x+\sum_{s=1}^{K} C_{i s} y_{s} \leq b_{i}, j=1,2, \ldots, K,
\end{aligned}
$$

where $i=1,2, \ldots, K$, by Definition $2(\mathrm{a})$.

This simple transformation has shown that solving the linear BLMFP (1) is equivalent to solving (4a-f).

(2) Necessity is obvious from (4a-f).

(3) Sufficiency.

If $\left(x^{*}, y_{1}^{*}, \ldots, y_{K}^{*}\right)$ is the optimal solution of (1a-d), we need to show that there exist (row) vectors $u_{1}^{*}, u_{2}^{*}, \ldots, u_{K}^{*}, v_{1}^{*}, v_{2}^{*}, \ldots, v_{K}^{*}$ and $w_{1}^{*}, w_{2}^{*}, \ldots, w_{K}^{*}$ such that $\left(x^{*}, y_{1}^{*}, \ldots, y_{K}^{*}, u_{1}^{*}, \ldots, u_{K}^{*}\right.$, $\left.v_{1}^{*}, \ldots, v_{K}^{*}, w_{1}^{*}, \ldots, w_{K}^{*}\right)$ to solve (4a-f). Going one step farther, we only need to proof that there exist (row) vectors $u_{1}^{*}, u_{2}^{*}, \ldots, u_{K}^{*}, v_{1}^{*}, v_{2}^{*}, \ldots, v_{K}^{*}$ and $w_{1}^{*}, w_{2}^{*}, \ldots, w_{K}^{*}$ such that $\left(x^{*}, y_{1}^{*}, \ldots, y_{K}^{*}, u_{1}^{*}, \ldots, u_{K}^{*}, v_{1}^{*}, \ldots, v_{K}^{*}, w_{1}^{*}, \ldots, w_{K}^{*}\right)$ satisfies the follows

$$
\begin{gathered}
u_{i} B_{i}+v_{i} C_{i}^{\prime}-w_{i}=-e_{i i} \\
u_{i}\left(b-A x-\sum_{s=1}^{K} B_{s} y_{s}\right)=0 \\
v_{i}\left(b^{\prime}-A^{\prime} x-\sum_{s=1}^{K} C_{s}^{\prime} y_{s}\right)=0 \\
w_{i} y_{i}=0
\end{gathered}
$$


where $u_{i} \in R^{p}, v_{i} \in R^{q_{1}+q_{2}+\ldots+q_{K}}, w_{i} \in R^{m_{i}}, i=1,2, \ldots, K$ and they are not negative variables. Because $\left(x^{*}, y_{1}^{*}, \ldots, y_{K}^{*}\right)$ is the optimal solution of $(1 \mathrm{a}-\mathrm{d})$, we have

$$
\left(x^{*}, y_{1}^{*}, \ldots, y_{K}^{*}\right) \in I R,
$$

by (2). Thus we have

$$
y_{i}^{*} \in P_{i}\left(x^{*}\right),
$$

where $i=1,2, \ldots, K$, by Definition 2(e). Consequently, $\left(y_{1}^{*}, y_{2}^{*}, \ldots, y_{K}^{*}\right)$ is the optimal solution to the following problem

$$
\min \left(f_{i}\left(x^{*}, y_{1}, \ldots, y_{K}\right): y_{i} \in S_{i}\left(x^{*}\right)\right),
$$

where $i=1,2, \ldots, K$, by Definition 2(d). Rewrite it as follows

$\min f_{i}\left(x, y_{1}, \ldots, y_{K}\right)$

subject to $y_{i} \in S_{i}(x)$

$$
\begin{aligned}
& x=x^{*} \\
& y_{j}=y_{j}^{*}, j=1,2, \ldots, K, j \neq i,
\end{aligned}
$$

where $i=1,2, \ldots, K$. From Definition 2(c), we have

$$
\begin{gathered}
\min f_{i}\left(x, y_{1}, \ldots, y_{K}\right)=c_{i} x+\sum_{s=1}^{K} e_{i s} y_{s} \\
\text { subject to } A x+\sum_{s=1}^{K} B_{s} y_{s} \leq b \\
A_{j} x+\sum_{s=1}^{K} C_{j s} y_{s} \leq b_{j}, j=1,2, \ldots, K \\
x=x^{*} \\
y_{i} \geq 0 \\
y_{j}=y_{j}^{*}, j=1,2, \ldots, K, j \neq i
\end{gathered}
$$

where $i=1,2, \ldots, K$. Let us define:

$$
A^{\prime}=\left(A_{1}, A_{2}, \ldots, A_{K}\right)^{-1}, b^{\prime}=\left(b_{1}, b_{2}, \ldots, b_{K}\right)^{-1}, C_{i}^{\prime}=\left(C_{i 1}, C_{i 2}, \ldots, C_{i K}\right)^{-1}, i=1,2 \ldots, K \text {. Tо }
$$

simplify (6c), we can have 


$$
\begin{gathered}
\min f_{i}\left(x, y_{1}, \ldots, y_{K}\right)=c_{i} x+\sum_{s=1}^{K} e_{i s} y_{s} \\
\text { subject to } A x+\sum_{s=1}^{K} B_{s} y_{s} \leq b \\
A^{\prime} x+\sum_{s=1}^{K} C_{s}^{\prime} y_{s} \leq b^{\prime} \\
x=x^{*} \\
y_{i} \geq 0 \\
y_{j}=y_{j}^{*}, j=1,2, \ldots, K, j \neq i,
\end{gathered}
$$

where $i=1,2, \ldots, K$. Thus simplify it, we can have

$$
\begin{gathered}
\min f_{i}\left(y_{i}\right)=e_{i i} y_{i} \\
\text { subject to }-\left(\begin{array}{c}
B_{i} \\
C_{i}^{\prime}
\end{array}\right) y_{i} \geq-\left(\begin{array}{l}
b-A x^{*}-\sum_{s=1, s \neq i}^{K} B_{s} y_{s}^{*} \\
b_{i}^{\prime}-A^{\prime} x^{*}-\sum_{s=1, s \neq i}^{K} C_{s}^{\prime} y_{s}^{*}
\end{array}\right) \\
y_{i} \geq 0
\end{gathered}
$$

where $i=1,2, \ldots, K$.

Now we see that $y_{i}^{*}$ is the optimal solution of (7) which is a LP problem. By Proposition 1 , there exists vector $\lambda_{i}^{*}, \mu_{i}^{*}, i=1,2, \ldots, K$ that satisfy a system below

$$
\begin{aligned}
& \lambda_{i}\left(\begin{array}{l}
B_{i} \\
C_{i}^{\prime}
\end{array}\right)-\mu_{i}=-e_{i i} \\
& \lambda_{i}\left(-\left(\begin{array}{l}
B_{i} \\
C_{i}^{\prime}
\end{array}\right) y_{i}+\left(\begin{array}{l}
b-A x^{*}-\sum_{s=1, s \neq i}^{K} B_{s} y_{s}^{*} \\
b^{\prime}-A^{\prime} x^{*}-\sum_{s=1, s \neq i}^{K} C_{s}^{\prime} y_{s}^{*}
\end{array}\right)=0\right. \\
& \mu_{i} y_{i}=0,
\end{aligned}
$$

where $\lambda_{i} \in R^{p+q_{1}+\ldots+q_{K}}, \mu_{i} \in R^{m_{i}}, i=1,2, \ldots, K$.

Let $u_{i} \in R^{p}, v_{i} \in R^{q_{i}+q_{2}+\ldots+q_{K}}, w_{i} \in R^{m_{i}}$ and define 


$$
\begin{aligned}
& \lambda_{i}=\left(u_{i}, v_{i}\right) \\
& w_{i}=\mu_{i},
\end{aligned}
$$

where $i=1,2, \ldots, K$. Thus we have $\left(x^{*}, y_{1}^{*}, \ldots, y_{K}^{*}, u_{1}^{*}, \ldots, u_{K}^{*}, v_{1}^{*}, \ldots, v_{K}^{*}, w_{1}^{*}, \ldots, w_{K}^{*}\right)$ that satisfy (8a-n). Our proof is completed.

Theorem 2 means that the most direct approach to solving (1a-d) is to solve the equivalent mathematical program given in (7a-c). One advantage that it offers is that it allows for a more robust model to be solved without introducing any new computational difficulties.

\section{A numeric example}

\section{Example 1}

Consider a following linear BLMF problem with $x_{1}, x_{2} \in R^{1}, y_{1}, y_{2} \in R^{1}, z \in R^{1}$ and

$$
\begin{gathered}
X=\left\{x_{1} \geq 0, x_{2} \geq 0\right\}, Y=\left\{y_{1} \geq 0, y_{2} \geq 0, y_{3} \geq 0\right\}, \\
\min _{x_{1} \in X, x_{2} \in X} F\left(x_{1}, x_{2}, y_{1}, y_{2}, z\right)=-8 x_{1}-4 x_{2}-4 y_{1}+40 y_{2}+4 y_{3} \\
\text { subject to } 2 x_{1}-y_{1}+2 y_{2}-0.5 y_{3} \leq 1 \\
\min _{y_{1} \in Y} f_{1}\left(x_{1}, x_{2}, y_{1}, y_{2}, z\right)=2 x_{1}+x_{2}+2 y_{1}-y_{2}-y_{3} \\
\min _{y_{2} \in Y} f_{2}\left(x_{1}, x_{2}, y_{1}, y_{2}, z\right)=x_{1}+2 x_{2}-y_{1}+2 y_{2}-y_{3} \\
\min _{y_{3} \in Y_{3}} f_{3}\left(x_{1}, x_{2}, y_{1}, y_{2}, z\right)=3 x_{1}+3 x_{2}+y_{1}+y_{2}-2 y_{3} \\
\text { subject to } 2 x_{2}+2 y_{1}-y_{2}-0.5 y_{3} \leq 1 \\
\quad-y_{1}+y_{2}+y_{3} \leq 1 .
\end{gathered}
$$

Let us give Example 1 to show how the Kuhn-Tucker approach works. According to our approach, let us write all the inequalities but $x_{1} \geq 0, x_{2} \geq 0, y_{3} \geq 0$ of the transferred form of Example 1 as follows:

$$
\begin{aligned}
& g_{u, 1}\left(x_{1}, x_{2}, y_{1}, y_{2}, y_{3}\right)=1-\left(2 x_{1}-y_{1}+2 y_{2}-0.5 y_{3}\right) \geq 0 \\
& g_{v, 1}\left(x_{1}, x_{2}, y_{1}, y_{2}, y_{3}\right)=1-\left(2 x_{2}+2 y_{1}-y_{2}-0.5 y_{3}\right) \geq 0 \\
& g_{v, 2}\left(x_{1}, x_{2}, y_{1}, y_{2}, y_{3}\right)=1-\left(-y_{1}+y_{2}+y_{3}\right) \geq 0
\end{aligned}
$$




$$
\begin{aligned}
& g_{w 1,1}\left(x_{1}, x_{2}, y_{1}, y_{2}, y_{3}\right)=y_{1} \geq 0 \\
& g_{w 2,1}\left(x_{1}, x_{2}, y_{1}, y_{2}, y_{3}\right)=y_{2} \geq 0 \\
& g_{w 3,1}\left(x_{1}, x_{2}, y_{1}, y_{2}, y_{3}\right)=y_{3} \geq 0 .
\end{aligned}
$$

From (3a-f), we have

$$
\begin{gathered}
\min \left(-8 x_{1}-4 x_{2}-4 y_{1}+40 y_{2}+4 y_{3}\right) \\
\text { subject to } 2 x_{1}-y_{1}+2 y_{2}-0.5 y_{3} \leq 1 \\
2 x_{2}+2 y_{1}-y_{2}-0.5 y_{3} \leq 1 \\
-y_{1}+y_{2}+y_{3} \leq 1 \\
-u_{11}+2 v_{11}-v_{12}-w_{11}=-2 \\
2 u_{21}-v_{21}+v_{22}-w_{21}=-2 \\
-0.5 u_{31}-0.5 v_{31}+v_{32}-w_{31}=2 \\
g_{u, 1} u_{11}+g_{v, 1} v_{11}+g_{v, 2} v_{12}+g_{w 1,1} w_{11}=0 \\
g_{u, 1} u_{21}+g_{v, 1} v_{21}+g_{v, 2} v_{22}+g_{w 2,1} w_{21}=0 \\
g_{u, 1} u_{31}+g_{v, 1} v_{31}+g_{v, 2} v_{32}+g_{w 3,1} w_{31}=0 \\
x_{1} \geq 0, x_{2} \geq 0, y_{1} \geq 0, y_{2} \geq 0, y_{3} \geq 0 \\
u_{11} \geq 0, v_{11} \geq 0, v_{12} \geq 0, w_{11} \geq 0 \\
u_{21} \geq 0, v_{21} \geq 0, v_{22} \geq 0, w_{21} \geq 0 \\
u_{31} \geq 0, v_{31} \geq 0, v_{32} \geq 0, w_{31} \geq 0 .
\end{gathered}
$$

From $(8 \mathrm{e}),(8 \mathrm{f}),(8 \mathrm{~g}),(81),(8 \mathrm{~m})$ and $(8 \mathrm{n})$, we can have following six possibilities.

Case 1: $\left(u_{11}, v_{11}, v_{12}, w_{11}, u_{21}, v_{21}, v_{22}, w_{21}, u_{31}, v_{31}, v_{32}, w_{31}\right)=(2,0,0,0,0,2,0,0,0,0,2,0)$

Case 2: $\left(u_{11}, v_{11}, v_{12}, w_{11}, u_{21}, v_{21}, v_{22}, w_{21}, u_{31}, v_{31}, v_{32}, w_{31}\right)=(0,0,2,0,0,2,0,0,0,0,2,0)$

Case 3: $\left(u_{11}, v_{11}, v_{12}, w_{11}, u_{21}, v_{21}, v_{22}, w_{21}, u_{31}, v_{31}, v_{32}, w_{31}\right)=(0,0,0,2,0,2,0,0,0,0,2,0)$

Case 4: $\left(u_{11}, v_{11}, v_{12}, w_{11}, u_{21}, v_{21}, v_{22}, w_{21}, u_{31}, v_{31}, v_{32}, w_{31}\right)=(2,0,0,0,0,0,0,2,0,0,2,0)$

Case 5: $\left(u_{11}, v_{11}, v_{12}, w_{11}, u_{21}, v_{21}, v_{22}, w_{21}, u_{31}, v_{31}, v_{32}, w_{31}\right)=(0,0,2,0,0,0,0,2,0,0,2,0)$ 
Case 6: $\left(u_{11}, v_{11}, v_{12}, w_{11}, u_{21}, v_{21}, v_{22}, w_{21}, u_{31}, v_{31}, v_{32}, w_{31}\right)=(0,0,0,2,0,0,0,2,0,0,2,0)$

From Case1, (8h), (8i), (8j) and (8k), we have

$$
\begin{aligned}
& g_{u, 1}\left(x_{1}, x_{2}, y_{1}, y_{2}, y_{3}\right)=1-\left(2 x_{1}-y_{1}+2 y_{2}-0.5 y_{3}\right)=0 \\
& g_{v, 1}\left(x_{1}, x_{2}, y_{1}, y_{2}, y_{3}\right)=1-\left(2 x_{2}+2 y_{1}-y_{2}-0.5 y_{3}\right)=0 \\
& g_{v, 2}\left(x_{1}, x_{2}, y_{1}, y_{2}, y_{3}\right)=1-\left(-y_{1}+y_{2}+y_{3}\right)=0 .
\end{aligned}
$$

Consequently, (8) can be rewritten as follows:

$$
\begin{aligned}
& \min \left(-8 x_{1}-4 x_{2}-4 y_{1}+40 y_{2}+4 y_{3}\right) \\
& \text { subject to } 2 x_{1}-y_{1}+2 y_{2}-0.5 y_{3}=1 \\
& \qquad x_{2}+2 y_{1}-y_{2}-0.5 y_{3}=1 \\
& \quad-y_{1}+y_{2}+y_{3}=1 \\
& \quad x_{1} \geq 0, x_{2} \geq 0, y_{1} \geq 0, y_{2} \geq 0, z \geq 0 .
\end{aligned}
$$

Using the simplex algorithm [2], we found that a solution occurs at the point $\left(x_{1}^{1}, x_{2}^{1}, y_{1}^{1}, y_{2}^{1}, y_{3}^{1}\right)=(1.5,0,1,0,2)$ with $F^{1}=-8, f_{1}^{1}=3, f_{2}^{1}=-1.5$ and $f_{3}^{1}=1.5$

By using the same way as that of Case 1, we have that a solution occurs at the point $\left(x_{1}^{2}, x_{2}^{2}, y_{1}^{2}, y_{2}^{2}, y_{3}^{2}\right)=(1.5,0,1,0,2)$ with $F^{2}=-8, f_{1}^{2}=3, f_{2}^{2}=-1.5$ and $f_{3}^{2}=1.5$ for Case 2; a solution occurs at the point $\left(x_{1}^{3}, x_{2}^{3}, y_{1}^{3}, y_{2}^{3}, y_{3}^{3}\right)=(0.75,0.75,0,0,1)$ with $F^{3}=-5$, $f_{1}^{3}=1.25, f_{2}^{3}=1.25$ and $f_{3}^{3}=2.5$ for Case 3 ; a solution occurs at the point $\left(x_{1}^{4}, x_{2}^{4}, y_{1}^{4}, y_{2}^{4}, y_{3}^{4}\right)=(1.5,0,1,0,2)$ with $F^{4}=-8, f_{1}^{4}=3, f_{2}^{4}=-1.5$ and $f_{3}^{4}=1.5$ for Case 4; a solution occurs at the point $\left(x_{1}^{5}, x_{2}^{5}, y_{1}^{5}, y_{2}^{5}, y_{3}^{5}\right)=(1.5,0,1,0,2)$ with $F^{5}=-8$, $f_{1}^{5}=3, f_{2}^{5}=-1.5$ and $f_{3}^{5}=1.5$ for Case 5 ; a solution occurs at the point $\left(x_{1}^{6}, x_{2}^{6}, y_{1}^{6}, y_{2}^{6}, y_{3}^{6}\right)=(0.75,0.75,0,0,0) \quad$ with $\quad F^{6}=-5, f_{1}^{6}=1.25, f_{2}^{6}=1.25 \quad$ and $f_{3}^{6}=2.5$ for Case 6 .

By examining above procedure, we found that the solution occurs at the point $\left(x_{1}^{*}, x_{1}^{*}, y_{1}^{*}, y_{2}^{*}, z^{*}\right)=(1.5,0,1,0,2)$ with $F^{*}=-8, f_{1}^{*}=3, f_{2}^{*}=-1.5, f_{3}^{*}=1.5$ for this Example 1. 


\section{Further remark}

Different relationships among followers in a BLMF decision problem could cause multiple different processes for deriving an optimal solution for the upper level's decision making. The referential-uncooperative situation is one of the popular cases in BLMF decision practices. For solving such a BLMF decision problem, this paper extended the Kuhn-Tucker approach from dealing with one leader and one follower to dealing with referential-uncooperative multiple followers. This paper further illustrated the details of the proposed approach by a numeric example. Initial experiment results showed this new extended approach more effectively for solving the proposed BLMF decision problem. Like most really powerful ideas, the basic notion of Nash equilibrium is very simple, even obvious. Its mathematical extensions and implications are not, however. The idea of this natural "sticking point" is that no single player can benefit from unilaterally changing his or her move - a non-cooperative best-response equilibrium [25]. As a future research, we are going to explore how use this concept into our BLMF research. Some practical use of this extended algorithm also will be considered as our future research task for BLMF decision making in the referential-uncooperative situation.

\section{References}

[1] H. V. Stackelberg, The Theory of the Market Economy: Oxford University Press, 1952.

[2] J. Bard, Practical bilevel optimization: algorithms and applications: Kluwer Academic Publishers, Boston, 1998.

[3] W. Candler and R. Townsley, "A linear twolevel programming problem," Computers and Operations Research, vol. 9, pp. 59-76, 1982.

[4] W. Bialas and M. Karwan, "Two-level linear programming," Management Science, vol. 30, pp. 1004-1020, 1984.

[5] J. Bard and J. Falk, "An explicit solution to the multilevel programming problem," Computers and Operations Research, vol. 9, pp. 77-100, 1982.

[6] W. Bialas and M. Karwan, "Multilevel linear programming," State University of New York at Buffalo, Technical Report 781, 1978.

[7] P. Hansen, B. Jaumard, and G. Savard, "New branchandbound rules for linear bilevel programming.," SIAM Journal on Scientific and Statistical Computing, vol. 13, pp. 1194-1217, 1992. 
[8] W. Bialas, M. Karwan, and J. Shaw, "A parametric complementary pivot approach for twolevel linear programming," State University of New York at Buffalo, Technical Report 802, 1980.

[9] E. Aiyoshi and K. Shimizu, "Hierarchical decentralized systems and its new solution by a barrier method," IEEE Transactions on Systems, Man, and Cybernetics, vol. 11, pp. 444-449, 1981.

[10] D. White and G. Anandalingam, "A penalty function approach for solving bilevel linear programs," Journal of Global Optimization, vol. 3, pp. 397-419, 1993.

[11] S. Scholtes and M. Stöhr, "Exact penalization of mathematical programs with equilibrium costraints," SIAM J. Control and Optimization, vol. 37, pp. 617-652, 1999.

[12] P. Marcotte and D. Zhu, "Exact and inexact penalty methods for the generalized bilevel programming problem," Mathematical Programming, vol. 74, pp. 142157, 1996.

[13] J. J. Ye and D. L. Zhu, "Exact penalization and necessary optimality conditions for generalized bilevel programming problems," SIAM J. Optim., vol. 7, pp. 481$507,1997$.

[14] A. Migdalas, "Bilevel programming in traffic planning: Models, methods and challenge," Journal of Global Optimisation, vol. 7, pp. 381-405, 1995.

[15] A. Migdalas, P. M. Pardalos, and P. Värbrand, Multilevel optimization-Algorithms and application: Kluwer Academics, 1997.

[16] P. Marcotte, "Network design problem with congestion effects: a case of bilevel programming," Mathematical Programming, vol. 34, pp. 142-162, 1986.

[17] C. Shi, G. Zhang, and J. Lu, "On the definition of linear bilevel programming solution," Applied Mathematics and Computation, vol. 160, pp. 169-176, 2005.

[18] C. Shi, J. Lu, and G. Zhang, "An extended Kth-best approach for linear bilevel programming," Applied Mathematics and Computation (in press), 2004.

[19] C. Shi, J. Lu, and G. Zhang, "An extended Kuhn-Tucker approach for linear bilevel programming," Applied Mathematics and Computation, vol. 162, pp. 51$63,2005$. 
[20] C. Shi, J. Lu, and G. Zhang, "An extended branch and bound algorithm for linear bilevel programming," (Submitted to )Applied Mathematics and Computation, 2004.

[21] J. Lu, C. Shi, and G. Zhang, "On bilevel multi-follower decision-making (I): general framework and solutions," (Accepted by) Information Sciences, 2005.

[22] C. Shi, G. Zhang, and J. Lu, "A Kth-best approach for linear bilevel multifollower programming," Journal of Global Optimization (In press), 2004.

[23] C. Shi, G. Zhang, and J. Lu, "A branch and bound algorithm for linear bilevel multi-follower programming," submitted to Journal of Computational Optimization and Application, 2004.

[24] "Heine-Borel theorem," http://thesaurus.maths.org/dictionary/map/word/10037 2003.

[25] "A brief Introduction to non- cooperative game theory," http://www.rh.edu/ stodder/BE/IntroGameT.htm 2005. 